\title{
On adsorption-induced denaturation of folded proteins
}

\author{
Ariel Fernández ${ }^{1}$ and Jeremy J. Ramsden ${ }^{2}$ \\ ${ }^{1}$ Instituto de Matemática, Universidad Nacional del Sur, Consejo Nacional de Investigaciones Cientificas y Técnicas, \\ Avenida Alem 1253, Bahía Blanca 8000, Argentina \\ ${ }^{2}$ Department of Biophysical Chemistry, Biozentrum, University of Basel, Switzerland
}

\begin{abstract}
In the absence of intramolecular long range interactions, the extended local conformation of a peptide chain is entropically favoured over compact ones, since the area of the Ramachandran basin of attraction is larger for the extended local conformation than for any other. In consequence, a random coil is predominantly in the extended conformation. Under refolding conditions, the high entropic content of the random coil is countered by the enthalpy loss associated with intramolecular contact formation ("hydrophobic collapse") and compactification of the chain. At an appropriate protein-binding surface, however, the enthalpy loss can be achieved at minimal cost of conformational entropy, since contacts can be formed between the protein and the surface and hence do not require compactification of the protein chain. This saving in entropic cost is a central concept for understanding the surface-induced denaturation of folded proteins.
\end{abstract}

Keywords: adsorption, conformation, denaturation, folding, protein

\section{INTRODUCTION AND BASIC TENETS OF THE THEORY}

All the local (Lennard-Jones, torsional and local dipole-dipole) contributions to the intramolecular potential for a folding protein are encapsulated in the so-called Ramachandran plots [1], plots of energy versus the two torsional dihedral angles $\phi$ and $\psi$ for each amino acid residue. The plots are divided into regions (basins), according to conformational type $[2,3]$. Each such plot may be viewed as a potential energy surface mapping the local $(\phi, \psi)$-torsional space for each residue onto the energy axis, i.e. directing the geometric constraints imposed on the protein backbone by the side chain torsional hindrances. The main topographical features of these maps are known to be invariant throughout the folding process, that is, even when the long range interactions set in $[3,4]$.

\footnotetext{
*Author for correspondence.

Collegium Basilea (Institute of Advanced Study), Hochstrasse 51, 4053 Basel, Switzerland.

e-mail: J.Ramsden@unibas.ch
}

The entropic content of a residue in the extended isomeric (rotameric) state is simply $\Delta S=R \ln P$, where $P=A_{\text {ext }} /(2 \pi)^{2}$ is the probability of a residue being in the extended conformation, $A_{\text {ext }}$ is the area of the extended basin and $4 \pi^{2}$ is the total area of the $\phi, \psi$ torus. A key observation is that the area of the basin of attraction corresponding to the local extended conformation is invariably larger than that for the locally compact torsional isomers. The basin areas of each of the twenty amino acids can be empirically estimated from a distribution of the $\phi, \psi$-coordinates plotted for a statistical sample of folded protein structures $[3,4]$. A point analysis of the plotted $(\phi, \psi)$ density coordinates obtained using the program PROCHECK gives an estimation of the relative areas (Table 1 gives results obtained with a statistical sample of 163 proteins). Direct inspection of this table reveals that in a random coil, regardless of its amino acid composition, the protein chain will adopt the locally extended conformation with the highest probability. Figure 1 shows a sketch of the $\phi, \psi-$ torus for a typical residue.

The large entropic content of the locally extended random coil is countered under renaturation conditions, when the protein forms intramolecular contacts at the expense of decreasing its entropy by adopting less probable local conformational states (Ramachandran basins 2 and 3), ultimately reaching the (marginally stable) folded state.

Now suppose we introduce a protein-binding surface whose affinity for the protein is appropriately 
Table 1. Areas of Ramachandran basins.

\begin{tabular}{ccccc}
\hline Residue & Basin 1 & Basin 2 & Basin 3 & Basin 4 \\
\hline ala & 55 & 41 & 4 & \\
arg & 52 & 42 & 6 & \\
asn & 46 & 36 & 18 & \\
asp & 50 & 42 & 8 & \\
cys & 50 & 45 & 5 & \\
gln & 48 & 43 & 9 & \\
glu & 53 & 41 & 6 & \\
gly & 26 & 24 & 30 & 20 \\
his & 46 & 42 & 12 & \\
ile & 56 & 42 & 2 & \\
leu & 54 & 42 & 4 & \\
lys & 51 & 42 & 7 & \\
met & 55 & 40 & 5 & \\
phe & 55 & 41 & 4 & \\
pro & 51 & 49 & 0 & \\
PP & 78 & 0 & 22 & \\
ser & 56 & 38 & 6 \\
thr & 51 & 46 & 3 \\
trp & 52 & 44 & 4 \\
tyr & 54 & 40 & 6 \\
val & 56 & 42 & 2 \\
\hline
\end{tabular}

Areas are given as percentages of $4 \pi^{2}$. These basins are known to exist for: L-alanyl-like residues (basins 1,2 and 3) (depicted in Fig. 1); glycine (basins 1,2,3 and 4); proline (basins 1 and 2); and (PP) any residue other than glycine or proline preceding proline (basins 1 and 3). Proline preceding proline resides solely in basin 1 .

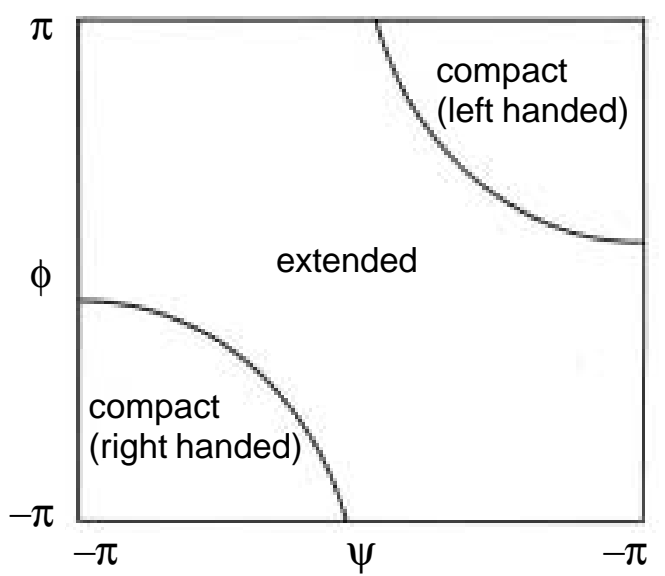

Figure 1. Sketch of the partition of the Ramachandran map. The basins of attraction represent local topological classes for an L-alanyl-like residue. The precise location of the separatrices (solid lines) is currently unknown, but the extended basin (1) invariably has a larger area than the compact regions (2,3 and 4$)$, i.e. $A_{\text {ext }}>A_{\text {com. }}$.

(by selecting suitable chemical functionalities [5]) tuned to achieve the same enthalpic loss as that of the intramolecularly folded protein. Calorimetric measurements show significant adsorption enthalpies for adsorbed proteins [6]. This should lead to adsorption-induced denaturation, since binding of the protein to the surface does not entail the entropic cost of relinquishing the highly probable extended basin in the Ramachandran maps. In other words, since the enthalpy loss associated with protein adsorption is not now countered by an entropic penalty, adsorption-induced denaturation will occur. In support of this assertion, there is experimental evidence for loss of secondary structure upon protein adsorption, ranging from almost negligible for cytochrome $\mathrm{c}$ to severe for serum albumin [7].

The goal of this paper is an analysis of the adsorption-induced denaturation. To the best of our knowledge, a semiempirical model rooted in entropic estimations of local available areas has not been attempted until now.

\section{ADSORPTION-INDUCED DENATURATION}

In developing a semiempirical model for adsorption-induced conformational changes, we focus initially on the simplest case: a chain forming a single intramolecular loop in the proximity of an adsorbing surface (Fig. 2). The entropy loss

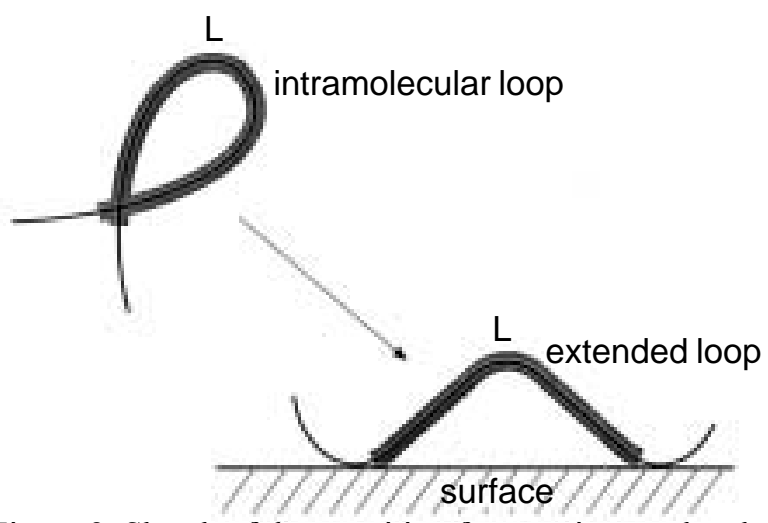

Figure 2. Sketch of the transition from an intramolecular compact to an adsorbed extended loop, each comprising $L$ residues.

associated with intramolecular loop formation is proportional to the logarithm of the ratio of the number $\Omega_{\text {loop }}$ of torsional looped conformations to the total number $\Omega$ of conformations available in the random coil. The loop sizes are considered to be small enough so as not to impose significant excludedvolume effects. Thus, the entropy loss contribution associated with $L$-loop formation scaling with $\ln L$ will be neglected. We assume that our system consists of protein and surface, with the effect of the solvent subsumed in the Ramachandran plots evaluated in Table 1, and in the effective hydrophobic forces, which translate the net entropic gain of solvent reorganization arising when two hydrophobic groups come in contact [8]. In the following discussion it is not necessary to explicitly quantify the net enthalpic 
change due to these forces when protein-surface contacts are substituted for protein-protein ones since the surface can be tuned to make $\Delta \Delta H=\Delta H_{\text {prot-surf }}-\Delta H_{\text {intrafold }}=0$, where $\Delta H_{\text {prot-surf }}$ is the enthalpy change associated with protein adsorption, and $\Delta H_{\text {intrafold }}$ is the net enthalpy change associated with intramolecular folding.

The multiplicity quotient $\Omega_{\text {loop }} / \Omega$ may be estimated by taking the restrictions (enshrined in the Ramachandran maps) imposed upon the local dynamics into account as the intramolecular loop is formed. Since there are two soft-mode torsional degrees of freedom $(\phi, \psi)$ per residue, and the random coil imposes no conformational restrictions, the area available to each residue in the random coil is $(2 \pi)^{2}$ and thus is simply proportional to $(2 \pi)^{2 N}$ for a chain containing $N$ residues. For a loop of $L$ residues, $\Omega_{\text {loop }}$ is proportional to $(2 \pi)^{2(N-L)} A_{c}^{L}$, where $A_{c}$ is the area of the basin region on the Ramachandran map corresponding to the conformation type $c$ of the loop: formation of the loop imposes local conformational constraints forcing each of the $L$ residues to reside in a basin corresponding to a local compact conformation. Our analysis is a topological one in that we do not distinguish between specific features of each basin, but focus only on the local conformational compatibility with a given secondary structural motif; for example, a right-handed helix turn, a $\beta$-bend or loop belong locally to the same topology class (a $\beta$-bend could be regarded as an $\alpha$-helix turn with zero pitch). We assume that local equilibration within Ramachandran basins is associated with shorter timescales than those needed for transitions between basins [2].

It follows that

$\Delta S_{\text {loop }}=R \ln \left(\frac{A_{\text {com }}}{(2 \pi)^{2}}\right)^{L}$.

where com designates the Ramachandran compact region compatible with intramolecular loop formation. The kinetic barrier for intramolecular contact formation is $B=-T \Delta S_{\text {loop }}$. There are two compact regions for an L-alanyl-like residue (the right and left-handed $\alpha$-helices), three for glycine, and only one for other types of residues [1,2]. Their extents are not well established, although as mentioned above the region of extended conformations is known to be substantially greater than the regions of compact local conformations (Table 1 and Fig. 1). The dearth of data on the local torsional parameters is due to the fact that the primary tool of analysis in the field is molecular dynamics [9], which gives virtually no information on the location of the separatrices (saddle maxima connexions) for each basin of attraction on the Ramachandran map.

At a critical denaturation temperature $T^{*}, \Delta H$ equals $T \Delta S_{\text {loop }}$ and the free energy difference between the folded and structureless conformations is zero.

Hence

$T_{\text {com }}^{*}=-\Delta H /\left[R L \ln \left(\frac{(2 \pi)^{2}}{A_{\text {com }}}\right)\right]$.

\section{RELATIVE BOLTZMANN AND KINETIC WEIGHTS OF FOLDED VERSUS ADSORBED STATES}

The adsorbed state enables the possibility of lowering the enthalpic content at the expense of minimal loss in conformational entropy [10] to be realized more advantageously compared with the intramolecular looped state. This assertion follows from our previous considerations once we compute the entropic cost of making an extended loop (Fig. 2), which has to fulfill two conditions:

(a) the extended loop involves the same residues previously involved in an intramolecular loop, but now one internal contact is replaced by two chainsurface contacts, with the same overall enthalpy loss presumed, since we can always find a surface for which it is the same (the protein-surface contact enthalpy can be tuned by modifying the surface charge, hydrophobicity, etc. [5]);

(b) all the residues in the adsorbed loop are in the entropically most favourable (extended) configuration. The main issue is then to compare the relative stabilities for both extended loop and intramolecular loop configurations. The entropy loss involved in preparing the extended loop state is estimated as (cf. eqn 1)

$\Delta S_{\mathrm{ext}}=R \ln \left(\frac{A_{\mathrm{ext}}}{(2 \pi)^{2}}\right)^{L}$.

The corresponding critical temperature $T^{*}{ }_{\text {ext }}$ is then $-\Delta H /\left[R L \ln \left\{(2 \pi)^{2} / A_{\text {ext }}\right\}\right]$ (cf. eqn 2 ), and the unimolecular rate constant is proportional to $\exp \left(\Delta S_{\text {ext }} / R\right)$. Since $A_{\text {ext }}>A_{\text {com }}, T^{*}{ }_{\text {com }}<T^{*}{ }_{\text {ext }}$. Hence one should get denatured (i.e. irreversible) adsorption at the surface in the temperature interval $T^{*}{ }_{\text {com }}<T<T^{*}{ }_{\text {ext. }}$. However, below $T^{*}{ }_{\text {com }}$ adsorption would presumably not be irreversible, and an equilibrium should be established - tilted towards the adsorbed state. The actual computation of the energy barriers and the Boltzmann weights gives us a direct estimate of the equilibrium constant for the compact 
vs extended loop configurations. From eqns (1) and (3) we can obtain the equilibrium constant for $\Delta \Delta H=0$ (i.e. heat neither gained nor lost in the adsorption-denaturation of the folded state):

$$
\begin{aligned}
& K_{\text {com } \rightarrow \text { ext }}=\exp \left(\frac{-\Delta \Delta G^{0}}{R T}\right)= \\
& =\exp \left(\frac{T\left(\Delta S_{\text {ext }}-\Delta S_{\text {com }}\right)}{R T}\right)=\left(\frac{A_{\text {ext }}}{A_{\text {com }}}\right)^{L} .
\end{aligned}
$$

\section{CONCLUSIONS}

At least two régimes for adsorption can be identified:

(i) irreversible adsorption in the range $T^{*}{ }_{\text {com }}<T<T^{*}{ }_{\text {ext }}$;

(ii) reversible adsorption for $T<T^{*}$ com.

Within the reversible régime the equilibrium constant is determined by a microscopic theory taking local torsional parameters within the chain dynamics into account. The resulting expression enables hitherto inaccessible microscopic information (i.e. the actual size of the basin regions) to become available through direct phenomenonological observation of the adsorption régimes. By isolating the purely entropic advantage of forming the extended adsorbed loop we have been able to provide Boltzmann weights as well as unimolecular rate constants for the formation of compact and extended loop structures.

\section{REFERENCES}

1. G.N. Ramachandran and V. Sasisekharan. $A d v$. Prot. Chem. 23 (1968) 283.

2. C.R. Cantor and P.R. Schimmel. Biophysical chemistry (Freeman, San Francisco, 1980), Parts I, II and III.

3. A. Fernández. Phys. Rev. E 59 (1999) 5928.

4. J.M. Thornton, in Protein Folding, edited by T.E. Creighton, (Freeman, New York, 1992) 59-63.

5. J.J. Ramsden, D.J. Roush, D.S. Gill, R. Kurrat and R.C. Willson. J. Amer. Chem. Soc. 117 (1995) 8511.

6. T. Arai and W. Norde. Colloids Surf. 51 (1990) 493.

7. A. Kondo, S. Oku and K. Higashitani. J. Colloid Interface Sci. 143 (1991) 214.

8. A. Fernández and O. Sinanoglu. Biophys. Chem. 21 (1985) 163.

9. C.L. Brooks, M. Karplus and B.M. Pettitt. Adv. Chem. Phys. 71 (1988).

10.A. Fernández and A. Colubri. J. Math. Phys. 39 (1998) 3167. 